\title{
Front Matter: Volume 8510
}

, "Front Matter: Volume 8510," Proc. SPIE 8510, Earth Observing Systems XVII, 851001 (15 October 2012); doi: 10.1117/12.2008661

SPIE Event: SPIE Optical Engineering + Applications, 2012, San Diego, California, United States 


\section{PROCEEDINGS OF SPIE}

\section{Earth Observing Systems XVII}

James J. Butler

Xiaoxiong Xiong

Xingfa Gu

Editors

13-16 August 2012

San Diego, California, United States

Sponsored and Published by

SPIE 
The papers included in this volume were part of the technical conference cited on the cover and title page. Papers were selected and subject to review by the editors and conference program committee. Some conference presentations may not be available for publication. The papers published in these proceedings reflect the work and thoughts of the authors and are published herein as submitted. The publisher is not responsible for the validity of the information or for any outcomes resulting from reliance thereon.

Please use the following format to cite material from this book:

Author(s), "Title of Paper," in Earth Observing Systems XVII, edited by James J. Butler, Xiaoxiong Xiong, Xingfa GU, Proceedings of SPIE Vol. 8510 (SPIE, Bellingham, WA, 2012) Article CID Number.

ISSN: 0277-786X

ISBN: 9780819492272

Published by

SPIE

P.O. Box 10, Bellingham, Washington 98227-0010 USA

Telephone +1 3606763290 (Pacific Time) · Fax +1 3606471445

SPIE.org

Copyright (C) 2012, Society of Photo-Optical Instrumentation Engineers.

Copying of material in this book for internal or personal use, or for the internal or personal use of specific clients, beyond the fair use provisions granted by the U.S. Copyright Law is authorized by SPIE subject to payment of copying fees. The Transactional Reporting Service base fee for this volume is $\$ 18.00$ per article (or portion thereof), which should be paid directly to the Copyright Clearance Center (CCC), 222 Rosewood Drive, Danvers, MA 01923. Payment may also be made electronically through CCC Online at copyright.com. Other copying for republication, resale, advertising or promotion, or any form of systematic or multiple reproduction of any material in this book is prohibited except with permission in writing from the publisher. The CCC fee code is 0277-786X/12/\$18.00.

Printed in the United States of America.

Publication of record for individual papers is online in the SPIE Digital Library.

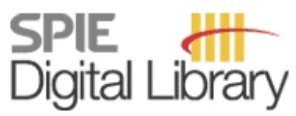

SPIEDigitalLibrary.org

Paper Numbering: Proceedings of SPIE follow an e-First publication model, with papers published first online and then in print and on CD-ROM. Papers are published as they are submitted and meet publication criteria. A unique, consistent, permanent citation identifier (CID) number is assigned to each article at the time of the first publication. Utilization of CIDs allows articles to be fully citable as soon as they are published online, and connects the same identifier to all online, print, and electronic versions of the publication. SPIE uses

a six-digit CID article numbering system in which:

- The first four digits correspond to the SPIE volume number.

- The last two digits indicate publication order within the volume using a Base 36 numbering system employing both numerals and letters. These two-number sets start with 00, 01, 02, 03, $04,05,06,07,08,09,0 \mathrm{~A}, 0 \mathrm{~B} \ldots \mathrm{OZ}$, followed by 10-1Z, 20-2Z, etc.

The CID number appears on each page of the manuscript. The complete citation is used on the first page, and an abbreviated version on subsequent pages. Numbers in the index correspond to the last two digits of the six-digit CID number. 


\section{Contents}

xi Conference Committee

SESSION 1 EARTH RADIATION BUDGET MISSIONS AND INSTRUMENTS

851002 ScaRaB: first results of the scanner for radiative budget on board the Indo-French satellite Megha-Tropiques [8510-1]

T. L. Trémas, N. Karouche, A. Rosak, A. Meygret, Ctr. National d'Études Spatiales (France);

O. Aznay, C-S (France); E. Hillairet, Magellium (France)

851003 On-orbit solar calibration methods using the Clouds and Earth's Radiant Energy System (CERES) in-flight calibration system [8510-2]

R. S. Wilson, Science Systems and Applications, Inc. (United States); K. J. Priestley, NASA Langley Research Ctr. (United States); S. Thomas, P. Hess, Science Systems and Applications, Inc. (United States)

851004 Performance stability of the Clouds and Earth's Radiant Energy System (CERES) instrument sensors on board the Aqua and Terra spacecraft [8510-3]

M. Shankar, Science Systems and Applications, Inc. (United States); K. Priestley, NASA Langley Research Ctr. (United States); S. Thomas, P. Hess, D. Walikainen, Science Systems and Applications, Inc. (United States)

851005 Performance of the Clouds and the Earth's Radiant Energy System (CERES) Flight Model 5 (FM5) instrument on NPP mission [8510-4]

S. Thomas, Science Systems and Applications, Inc. (United States); K. J. Priestley, NASA Langley Research Ctr. (United States); P. C. Hess, R. S. Wilson, N. P. Smith, M. G. Timcoe, M. Shankar, D. R. Walikainen, Science Systems and Applications, Inc. (United States)

851006 A strategy to assess the pointing accuracy of the CERES (FM5) scanner [8510-5] N. P. Smith, Z. P. Szewczyk, P. C. Hess, Science Systems and Applications, Inc. (United States); K. J. Priestley, NASA Langley Research Ctr. (United States)

\section{SESSION $2 \quad$ NEW MISSIONS AND INSTRUMENTS}

851007 Early orbit operations performance of the Suomi NPP OMPS instrument [8510-6] M. Kowalewski, Universities Space Research Association (United States) and NASA Goddard Space Flight Ctr. (United States); C. Pan, Univ. of Maryland, College Park (United States); S. Janz, NASA Goddard Space Flight Ctr. (United States)

$851008 \quad$ Next-generation pushbroom filter radiometers for remote sensing [8510-7] R. W. Tarde, M. G. Dittman, G. E. Kvaran, Ball Aerospace \& Technologies Corp. (United States) 
851009 Out of band scatter measurements from OLI optical bandpass filters [8510-8]

P. D. Fuqua, C. J. Panetta, J. D. Barrie, The Aerospace Corp. (United States); E. Waluschka, NASA Goddard Space Flight Ctr. (United States); T. A. Mooney, Materion Barr Precision Optics \& Thin Film Coatings (United States)

8510 OA Dark and background response stability for the Landsat 8 Thermal Infrared Sensor [8510-9] K. Vanderwerff, SGT, Inc. (United States); M. Montanaro, Sigma Space Corp. (United States)

$8510 \mathrm{OB}$ Instrumentation and first results of the reflected solar demonstration system for the Climate Absolute Radiance and Refractivity Observatory [8510-10] J. McCorkel, K. Thome, J. Hair, B. McAndrew, D. Jennings, D. Rabin, A. Daw, NASA Goddard Space Flight Ctr. (United States); A. Lunsford, NASA Goddard Space Flight Ctr. (United States) and Catholic Univ. of America (United States)

8510 OC Multislit optimized spectrometer for ocean color remote sensing [8510-11]

T. Valle, C. Hardesty, Ball Aerospace \& Technologies Corp. (United States); C. O. Davis, N. Tufillaro, Oregon State Univ. (United States); M. Stephens, W. Good, P. Spuhler, Ball Aerospace \& Technologies Corp. (United States)

\section{SESSION 3 PRE-LAUNCH CALIBRATION AND CHARACTERIZATION}

8510 OD The distance dependences and spatial uniformities of spectral irradiance standard lamps [8510-12]

H. W. Yoon, National Institute of Standards and Technology (United States);

G. D. Graham, ITT Geospatial Systems Division, LLC (United States); R. D. Saunders, Y. Zong,

E. L. Shirley, National Institute of Standards and Technology (United States)

$8510 \mathrm{OE}$ Validation of the dissemination of spectral irradiance values using FEL lamps [8510-13]

B. C. Johnson, National Institute of Standards and Technology (United States);

G. D. Graham, Exelis Inc. (United States); R. D. Saunders, H. W. Yoon, E. L. Shirley, National Institute of Standards and Technology (United States)

8510 OF Preferred mirror coatings for UV, visible, and IR space optical instruments [8510-14] J. B. Heaney, L. R. Kauder, S. C. Freese, SGT, Inc. (United States); M. A. Quijada, NASA Goddard Space Flight Ctr. (United States)

8510 0G New approach to spectral features modeling [8510-15]

H. van Brug, P. S. Scalia, TNO Delft (Netherlands)

\section{SESSION 4 ON-ORBIT CALIBRATION AND CHARACTERIZATION}

$8510 \mathrm{OH}$ On-orbit performance of MODIS solar diffuser stability monitor [8510-16]

$X$. Xiong, NASA Goddard Space Flight Ctr. (United States); A. Angal, Science Systems and Applications, Inc. (United States); T. Choi, J. Sun, Sigma Space Corp. (United States);

E. Johnson, Raytheon Space \& Airborne Systems (United States) 
8510 ol Recent progress of MODIS solar diffuser on-orbit degradation characterization [8510-17] H. Chen, Z. Wang, J. Sun, Sigma Space Corp. (United States); A. Angal, Science Systems and Applications, Inc. (United States); X. Xiong, NASA Goddard Space Flight Ctr. (United States)

8510 0J On-orbit performance of the MODIS reflective solar bands time-dependent response versus scan angle algorithm [8510-18]

J. Sun, Sigma Space Corp. (United States); X. Xiong, NASA Goddard Space Flight Ctr. (United States); A. Angal, Science Systems and Applications, Inc. (United States); H. Chen, X. Geng, A. Wu, Sigma Space Corp. (United States)

8510 OK Monitoring and assessment of the temperature fluctuation of Aqua MODIS cold focal plane assembly [8510-62]

Z. Wang, Sigma Space Corp. (United States); S. Madhavan, Science Systems and Applications, Inc. (United States); X. Xiong, NASA Goddard Space Flight Ctr. (United States); A. Wu, B. Wenny, Sigma Space Corp. (United States)

8510 OL On-board calibration radiance error correction for MetOp/AVHRR IR channel [8510-19] T. Chang, I. M. Systems Group, Inc. (United States); X. WU, NOAA/NESDIS/STAR (United States)

8510 OM Recent advances in calibration of the GOES Imager visible channel at NOAA [8510-21] C. Dean, I.-L. Chang, Earth Resources Technology, Inc. (United States); Z. Li, SGT, Inc. (United States); M. Weinreb, Riverside Technology, Inc., NOAA/NESDIS/OSD (United States); X. WU, NOAA/NESDIS/STAR (United States); F. Yu, Earth Resources Technology, Inc. (United States)

\section{SESSION 5 INSTRUMENT CROSS-COMPARISON AND VICARIOUS CALIBRATION}

8510 ON Cross-calibration of imaging sensors using model-based, SI-traceable predictions of ATsensor radiance [8510-22]

K. Thome, J. McCorkel, NASA Goddard Space Flight Ctr. (United States)

851000 A comparative study of stripe noise on MODIS and VIIRS thermal emissive bands [8510-23] M. Bouali, NOAA/NESDIS/STAR (United States) and Colorado State Univ. (United States); A. Ignatov, NOAA/NESDIS/STAR (United States)

8510 OP NPP VIIRS and Aqua MODIS RSB comparison using observations from simultaneous nadir overpasses (SNO) [8510-24]

A. Wu, Sigma Space Corp. (United States); X. Xiong, NASA Goddard Space Flight Ctr. (United States)

$85100 Q$ Using SCIAMACHY to improve corrections for spectral band differences when transferring calibration between visible sensors [8510-25]

B. Scarino, Science Systems and Applications, Inc. (United States); D. R. Doelling, NASA Langley Research Ctr. (United States); D. L. Morstad, R. Bhatt, A. Gopalan, Science Systems and Applications, Inc. (United States); C. Lukashin, P. Minnis, NASA Langley Research Ctr. (United States) 
8510 OR Refined algorithms for star-based monitoring of GOES Imager visible-channel responsivities [8510-26]

I.-L. Chang, C. Dean, Earth Resources Technology, Inc. (United States); Z. Li, SGT, Inc.

(United States); M. Weinreb, Riverside Technology, Inc., NOAA/NESDIS/OSD (United States);

X. WU, NOAA/NESDIS/STAR (United States); P. A. Swamy, Federal Reserve Board (United

States)

\section{SESSION 6 ATMOSPHERIC INFRARED SOUNDER}

8510 OS Relative trends of AIRS and IASI radiometric calibrations [8510-27]

D. A. Elliott, H. H. Aumann, Jet Propulsion Lab. (United States)

8510 OT Validation of the radiometric stability of the Atmospheric Infrared Sounder [8510-28]

H. H. Aumann, D. Elliott, Jet Propulsion Lab. (United States); L. L. Strow, Univ. of Maryland, Baltimore County (United States)

8510 OU Significant advances in the AIRS Science Team Version-6 retrieval algorithm [8510-29] J. Susskind, NASA Goddard Space Flight Ctr. (United States); J. Blaisdell, L. Iredell, SAIC, NASA Goddard Space Flight Ctr. (United States)

8510 OV Level-1C product from AIRS: principal component filtering [8510-30]

E. M. Manning, Y. Jiang, H. H. Aumann, D. A. Elliott, Jet Propulsion Lab. (United States);

S. Hannon, Univ. of Maryland, Baltimore County (United States)

\section{SESSION 7 REMOTE SENSING DATA ANALYSIS}

$85100 Z$ Monitoring sea ice based on NOAA/AVHRR data [8510-34]

J. Shi, J. Guo, T. Zheng, Q. Wang, J. Zhu, Nanjing Univ. of Information Science \& Technology (China)

\section{SESSION $8 \quad$ VIIRS I}

851010 Radiometric calibration of a $100 \mathrm{~cm}$ sphere integrating source for VIIRS solar diffuser stability monitor bands [8510-35]

E. D. Kim, V. Murgai, R. W. Menzel, Raytheon Space \& Airborne Systems (United States)

851011 Preliminary assessment of Suomi-NPP VIIRS on-orbit radiometric performance [8510-36]

H. Oudrari, J. McIntire, Sigma Space Corp. (United States); D. Moyer, The Aerospace Corp. (United States); K. Chiang, Sigma Space Corp. (United States); X. Xiong, J. Butler, NASA Goddard Space Flight Ctr. (United States)

851012 VIIRS day-night band gain and offset determination and performance [8510-37]

J. Geis, C. Florio, D. Moyer, K. Rausch, F. J. De Luccia, The Aerospace Corp. (United States) 
851013 NPP VIIRS early on-orbit geometric performance [8510-38]

R. E. Wolfe, NASA Goddard Space Flight Ctr. (United States); G. Lin, NASA Goddard Space Flight Ctr. (United States) and Innovim (United States); M. Nishihama, NASA Goddard Space Flight Ctr. (United States) and Sigma Space Corp. (United States); K. P. Tewari, NASA Goddard Space Flight Ctr. (United States) and Innovim (United States); E. Montano, NASA Goddard Space Flight Ctr. (United States) and Sigma Space Corp. (United States)

851014 SUomi NPP VIIRS SDR postlaunch calibration/validation: an overview of progress, challenges, and the way forward [8510-39]

C. Cao, National Oceanic and Atmospheric Administration (United States); X. Xiong, NASA Goddard Space Flight Ctr. (United States); F. Weng, National Oceanic and Atmospheric Administration (United States)

\section{SESSION 9 VIIRS II}

851015 Characterization and performance of the Suomi-NPP/VIIRS solar diffuser stability monitor [8510-40]

J. P. Fulbright, N. Lei, K. Chiang, Sigma Space Corp. (United States); X. Xiong, NASA

Goddard Space Flight Ctr. (United States)

851016 VIIRS solar diffuser bidirectional reflectance distribution function (BRDF) degradation factor operational trending and update [8510-41]

E. Haas, D. Moyer, F. De Luccia, K. Rausch, The Aerospace Corp. (United States);

J. Fulbright, Sigma Space Corp. (United States)

851017 Characterization of Suomi-NPP VIIRS reflective solar bands dual gain anomaly [8510-61]

S. Lee, J. McIntire, H. Oudrari, Sigma Space Corp. (United States)

851018 Initial on-orbit radiometric calibration of the Suomi NPP VIIRS reflective solar bands [8510-43]

N. Lei, Z. Wang, J. Fulbright, S. Lee, J. McIntire, K. Chiang, Sigma Space Corp. (United

States); X. Xiong, NASA Goddard Space Flight Ctr. (United States)

851019 Operational calibration of VIIRS reflective solar band sensor data records [8510-44]

J. C. Cardema, K. W. Rausch, The Aerospace Corp. (United States); N. Lei, Sigma Space

Corp. (United States); D. I. Moyer, F. J. De Luccia, The Aerospace Corp. (United States)

$85101 \mathrm{~A}$ Discovery and characterization of on-orbit degradation of the Visible Infrared Imaging Radiometer Suite (VIIRS) Rotating Telescope Assembly (RTA) [8510-46]

F. De Luccia, D. Moyer, The Aerospace Corp. (United States); E. Johnson, Raytheon Space

\& Airborne Systems (United States); K. Rausch, The Aerospace Corp. (United States); N. Lei,

K. Chiang, Sigma Space Corp. (United States); X. Xiong, NASA Goddard Space Flight Ctr.

(United States); J. Fulbright, Sigma Space Corp. (United States); E. Haas, The Aerospace

Corp. (United States); G. Iona, NASA Goddard Space Flight Ctr. (United States) 
8510 1B Root cause determination of on-orbit degradation of the VIIRS rotating telescope assembly [8510-47]

J. D. Barrie, P. D. Fuqua, M. J. Meshishnek, M. R. Ciofalo, C. T. Chu, J. A. Chaney,

R. M. Moision, The Aerospace Corp. (United States); L. Graziani, SGT, Inc. (United States)

8510 IC VIIRS on-orbit optical anomaly: investigation, analysis, root cause determination and lessons learned [8510-48]

G. Iona, J. Butler, NASA Goddard Space Flight Ctr. (United States); B. Guenther, Stellar Solutions, Inc. (United States); L. Graziani, SGT, Inc. (United States); E. Johnson, Raytheon Space \& Airborne Systems (United States); B. Kennedy, Kennedy Consulting, Inc. (United States); C. Kent, Stellar Solutions, Inc. (United States); R. Lambeck, MEl Technologies, Inc. (United States); E. Waluschka, X. Xiong, NASA Goddard Space Flight Ctr. (United States)

\section{SESSION 11 VIIRS IV}

8510 ID VIIRS thermal emissive bands calibration algorithm and on-orbit performance [8510-49] D. Moyer, The Aerospace Corp. (United States); J. McIntire, Sigma Space Corp. (United States); F. De Luccia, The Aerospace Corp. (United States); B. Efremova, K. Chiang, Sigma Space Corp. (United States); X. Xiong, NASA Goddard Space Flight Ctr. (United States)

8510 1E NPP VIIRS emissive band radiance calibration [8510-50]

Q. Liu, Univ. of Maryland (United States); C. Cao, F. Weng, NOAA/NESDIS/STAR/Satellite Meteorology and Climatology Div. (United States)

$8510 \mathrm{lF} \quad$ VIIRS emissive band radiometric performance trending [8510-51]

E. Johnson, C. Ranshaw, Raytheon Space \& Airborne Systems (United States)

\section{SESSION 12 VIIRS V}

$85101 G \quad$ VIIRS on-orbit calibration for ocean color data processing [8510-52]

R. E. Eplee, Jr., K. R. Turpie, G. F. Fireman, SAIC (United States); G. Meister, NASA Goddard Space Flight Ctr. (United States); T. C. Stone, U.S. Geological Survey (United States);

F. S. Patt, SAIC (United States); B. A. Franz, NASA Goddard Space Flight Ctr. (United States);

S. W. Bailey, FutureTech Corp. (United States); W. D. Robinson, SAIC (United States);

C. R. McClain, NASA Goddard Space Flight Ctr. (United States)

$85101 \mathrm{H} \quad$ SUomi NPP VIIRS ocean color data product early mission assessment [8510-53]

K. R. Turpie, W. D. Robinson, SAIC (United States); B. A. Franz, NASA Goddard Space Flight Ctr. (United States); R. E. Eplee, Jr., SAIC (United States); G. Meister, NASA Goddard Space Flight Ctr. (United States); G. F. Fireman, F. S. Patt, R. A. Barnes, SAIC (United States);

C. R. McClain, NASA Goddard Space Flight Ctr. (United States)

$851011 \quad$ NPP VIIRS on-orbit calibration and characterization using the moon [8510-54] J. Sun, Sigma Space Corp. (United States); X. Xiong, J. Butler, NASA Goddard Space Flight Ctr. (United States)

$85101 \mathrm{~J}$ An overview of Suomi NPP VIIRS calibration maneuvers [8510-55]

J. J. Butler, X. Xiong, NASA Goddard Space Flight Ctr. (United States); R. A. Barnes, F. S. Patt, SAIC (United States); J. Sun, K. Chiang, Sigma Space Corp. (United States) 
$85101 \mathrm{~K}$ Analysis of Suomi-NPP VIIRS vignetting functions based on yaw maneuver data [8510-56] J. McIntire, B. Efremova, Sigma Space Corp. (United States); D. Moyer, The Aerospace Corp. (United States); S. Lee, Sigma Space Corp. (United States); X. Xiong, NASA Goddard Space Flight Ctr. (United States)

$85101 \mathrm{~L}$ VIIRS VisNIR/SMWIR end of life sensitivity predictions [8510-57]

V. Murgai, N. Nelson, E. Johnson, K. Yokoyama, Raytheon Space \& Airborne Systems (United States)

POSTER SESSION

8510 iN Analog and digital saturation in the MODIS reflective solar bands [8510-59]

S. Madhavan, A. Angal, Science Systems and Applications, Inc. (United States); J. Dodd, J. Sun, Sigma Space Corp. (United States); X. Xiong, NASA Goddard Space Flight Ctr. (United States)

$85101 \mathrm{P}$ Evolving high fidelity climate sensor simulators to preserve climate data record continuity [8510-60]

K. K. Teague, G. L. Smith, Science Systems and Applications, Inc. (United States);

K. Priestley, NASA Langley Research Ctr. (United States)

$85101 Q$ Assessment of the NPP VIIRS RVS for the thermal emissive bands using the first pitch maneuver observations [8510-63]

A. Wu, Sigma Space Corp. (United States); X. Xiong, NASA Goddard Space Flight Ctr. (United States); K. Chiang, C. Sun, Sigma Space Corp. (United States)

8510 is Suomi NPP VIIRS spectral characterization: understanding multiple RSR releases [8510-66] C. Moeller, Univ. of Wisconsin-Madison (United States); J. McIntire, T. Schwarting, Sigma Space Corp. (United States); D. Moyer, The Aerospace Corp. (United States); J. Costa, MIT Lincoln Lab. (United States)

8510 IT On-orbit characterization of the GOES Imager channel-to-channel co-registration [8510-20]

M. G. Grotenhuis, Earth Resources Technology, Inc. (United States); X. Wu,

NOAA/NESDIS/STAR (United States); F. YU, Earth Resources Technology, Inc. (United States); T. J. Schmit, NOAA/NESDIS/STAR (United States); S. S. Lindstrom, Univ. of Wisconsin-Madison (United States); C. Cao, NOAA/NESDIS/STAR (United States)

Author Index 
Proc. of SPIE Vol. $8510851001-10$

Downloaded From: https://www.spiedigitallibrary.org/conference-proceedings-of-spie on 26 Apr 2023 Terms of Use: https://www.spiedigitallibrary.org/terms-of-use 


\section{Conference Committee}

Program Track Chair

Allen H.-L. Huang, University of Wisconsin-Madison (United States)

Conference Chairs

James J. Butler, NASA Goddard Space Flight Center (United States)

Xiaoxiong Xiong, NASA Goddard Space Flight Center (United States)

Xingfa Gu, Institute of Remote Sensing Applications (China)

Conference Program Committee

Philip E. Ardanuy, Raytheon Intelligence \& Information Systems (United States)

Robert A. Barnes, NASA Goddard Space Flight Center (United States)

Hal J. Bloom, Earth Resources Technology, Inc. (United States)

Jeffrey S. Czapla-Myers, College of Optical Sciences, The University of Arizona (United States)

Armin Doerry, Sandia National Laboratories (United States)

Mitchell D. Goldberg, National Oceanic and Atmospheric Administration (United States)

Joel McCorkel, NASA Goddard Space Flight Center (United States)

Thomas S. Pagano, Jet Propulsion Laboratory (United States)

Jeffery J. Puschell, Raytheon Space \& Airborne Systems (United States)

Carl F. Schueler, Orbital Sciences Corporation (United States)

Session Chairs

1 Earth Radiation Budget Missions and Instruments

Xiaoxiong Xiong, NASA Goddard Space Flight Center (United States)

2 New Missions and Instruments

Thomas S. Pagano, Jet Propulsion Laboratory (United States)

3 Pre-Launch Calibration and Characterization

Xingfa Gu, Institute of Remote Sensing Applications (China)

4 On-Orbit Calibration and Characterization

Joel McCorkel, NASA Goddard Space Flight Center (United States) 
5 Instrument Cross-Comparison and Vicarious Calibration

James J. Butler, NASA Goddard Space Flight Center (United States)

6 Atmospheric Infrared Sounder

Armin Doerry, Sandia National Laboratories (United States)

7 Remote Sensing Data Analysis

Xiaoxiong Xiong, NASA Goddard Space Flight Center (United States)

8 VIIRS I

Jeffery J. Puschell, Raytheon Space \& Airborne Systems (United States)

9 VIIRS II

Carl F. Schueler, Orbital Sciences Corporation (United States)

10 VIIRS III

James J. Butler, NASA Goddard Space Flight Center (United States)

11 VIIRS IV

Philip E. Ardanuy, Raytheon Intelligence \& Information Systems (United States)

12 VIIRS V

James J. Butler, NASA Goddard Space Flight Center (United States) 